quality evidence). Olanzapine probably reduces delayed and vomiting. It is unclear if efficacy differs between $5 \mathrm{mg}$ and $10 \mathrm{mg}$ doses. We cannot exclude the possibility that $5 \mathrm{mg}$ may be associated with a lower risk of somnolence and fatigue than $10 \mathrm{mg}$.

Conclusions There is moderate quality evidence that oral olanzapine increases freedom from nausea and vomiting in adults undergoing chemotherapy for solid tumours. There is uncertainty whether it increases serious adverse events. It may increase the likelihood of other adverse events and probably increases somnolence and fatigue. There is uncertainty about the relative benefits and harms of lower $(5 \mathrm{mg})$ and higher (10 mg) doses.

\section{EVALUATION OF EXISTING PRACTICE: DENOSUMAB FOR HYPERCALCAEMIA OF MALIGNANCY}

Emily Kavanagh, Elizabeth Woods, Jolene Brown, Felicity Dewhurst, Kerry Waterfield, Grace Rowley, Jonathan Pickard, Donna Wakefield. Palliative Medicine Registrars at Health Education North East

\subsection{6/bmjspcare-2019-ASP.167}

Background Hypercalcaemia of malignancy (HoM) is a burdensome problem affecting up to $44 \%$ of patients with advanced cancer. The standard of care is treatment with an intravenous bisphosphonate. Bisphosphonates are contraindicated in renal failure.

Denosumab is a monoclonal antibody that inhibits osteoclast differentiation and activity. Licensed for the prevention of skeletal related events in patients with bone metastases, Denosumab has been noted to cause hypocalcaemia in previously normocalcaemic patients. Denosumab is administered by subcutaneous injection and is safe in renal failure.

Ad hoc use of Denosumab for HoM has been observed in the palliative care setting.

We aimed to evaluate existing practice around the use of Denosumab for HoM by palliative medicine consultants in the North East of England.

Methods We constructed an online survey informed by a targeted literature review. The survey was disseminated electronically to all consultants in the North East regional palliative care physicians' group. An email reminder was sent after 7 weeks. The survey was closed after 8 weeks.

Results There were 28 respondents out of a possible 34. Eleven (39.3\%) had used Denosumab for HoM. Of these, all used Denosumab for bisphosphonate refractory hypercalcaemia. There was some experience where bisphosphonates were contraindicated due to renal impairment. One patient was administered Denosumab at home. Four respondents used Denosumab in repeated doses for the same patient. Eight respondents reported that Denosumab successfully reduced serum calcium; three reported 'sometimes'. Doses varied between $60 \mathrm{mg}$ and $120 \mathrm{mg}$.

All consulted with another specialty (oncology 90.9\%) prior to using Denosumab.

There was high variability in the duration to re-test serum calcium. Two respondents encountered hypocalcaemia. There were no other adverse effects reported.

Conclusions Our results describe regional experience in the use of Denosumab. There was a wide variation in dosing and monitoring practices. The development of evidence-based guidance is strongly recommended.

\section{WHAT OUTCOMES ARE IMPORTANT IN A DRUG TRIAL FOR CHRONIC BREATHLESSNESS AND ARE WE CAPTURING CHANGE?}

N Lovell, SN Etkind, S Bajwah, M Maddocks, IJ Higginson. Cicely Saunders Institute, Department of Palliative Care, Policy and Rehabilitation, King's College London

\subsection{6/bmjspcare-2019-ASP.168}

Background Chronic breathlessness is common, distressing and remains a challenging to assess and treat. New treatments are emerging, but must be evaluated using appropriate outcomes that reflect the concerns of people experiencing the symptom.

Aim To determine which outcomes are important to patients with chronic breathlessness, and the ability of outcome measurement tools to capture change during a drug treatment trial. Methods Mixed-methods analysis of a multi-centre randomised double-blind feasibility trial of mirtazapine (ISRCTN registration 33236160). Quantitative outcome measures: numerical rating scale (NRS) for worst breathlessness in the last 24 hours, and chronic respiratory questionnaire (CRQ) were paired to in-depth qualitative data from interviews exploring outcomes of importance within the trial. Qualitative data was analysed thematically and quantitative data analysed according to the standard guidance for each questionnaire. The combined analyses were then triangulated, drawing upon a model of total breathlessness.

Results Paired data were available for 22 of 64 participants in the trial (data collected August 2016-December 2017). 16/22 (73\%) were male; median (range) age 72 (57-85) years. Primary diagnoses were chronic obstructive pulmonary disease 10/22 (45\%), interstitial lung disease 9/22 (41\%), chronic heart failure $2 / 22(9 \%)$ and lung cancer $1 / 22$ (4\%). Participants described outcomes of importance encompassing physical, social, emotional and spiritual domains. For many, perceived changes described during qualitative interview were consistent with changes in both NRS and CRQ scores. This was despite some participants expressing difficulties aligning an experience of breathlessness when completing scale for scale-based questionnaires.

Conclusions The main concerns described by participants in a drug trial for chronic breathlessness cross multiple health domains supporting a model of total breathlessness and the inclusion of multi-domain measures within trials. The agreement between paired qualitative and quantitative data indicates that both the NRS and CRQ outcome measures capture changes described by participants appropriately, and are suitable to use in definitive trials.

\section{A CLUSTER RANDOMISED FEASIBILITY TRIAL (CRCT) TO TEST THE ROUTINE USE OF THE NEEDS ASSESSMENT TOOL: CANCER (NAT:C) IN PRIMARY CARE TO REDUCE UNMET PATIENT AND CARER NEEDS AND DETERMINE THE FEASIBILITY OF A DEFINITIVE TRIAL}

Miriam Johnson, Elvis Amoakwa, Victoria Allgar, John Blenkinsopp, David Currow, Amanda Farrin, Robbie Foy, Una MacLeod, David Meads, Alexandra Wright-Hughes, Joseph Clark. Wolfson Palliative Care Centre, University of Hull, University of York, University of Northumbria, University of Leeds

\subsection{6/bmjspcare-2019-ASP.169}

Background People with cancer commonly have distressing symptoms and unidentified palliative care needs. The Needs 